\title{
Supporting progress towards the post-2015 targets and regional tuberculosis elimination: a statement of intent from the third meeting of the Asian TB Experts Community
}

\section{To the Editor:}

The World Health Organization (WHO) is challenging all countries, both high- and low-incidence, to dramatically intensify efforts to meet bold new goals of reducing global tuberculosis (TB) deaths by $95 \%$ and the incidence by $90 \%$ (<10 cases per 100000 population) by 2035 [1]. This radical strategic change came from the recognition that the current strategy of passive case finding and directly observed therapy (DOT) is not sufficiently curbing the incidence of TB. The new strategy calls for a synergy of interventions to enable early case detection, systematic screening and prevention of TB in contacts (adults and children) and high-risk groups, such as people living with HIV or other immune depressing conditions, people with diabetes, patients receiving dialysis, patients preparing for organ or haematological transplantation, patients with silicosis, prisoners, healthcare workers, homeless individuals, illicit drug users and individuals in communal settings. At the end of 2014, the WHO released the first guidelines on the management of latent tuberculosis infection (LTBI). The guidelines provide evidence based guidance on practices for testing, treating and managing LTBI in infected individuals with the highest likelihood of progression to active disease [2]. Although primarily aimed at high-income or upper middle-income countries, with an estimated TB incidence rate of $<100$ per 100000 population, they represent an unprecedented gear change in the scaling up of TB prevention as a component of TB control programmes [2]. Figure 1 shows a hypothetical approach to prioritisation of high-risk groups.

The available evidence suggests that latent TB screening, if performed according to evidence and targeting the correct people, may reduce suffering, death and all the downstream consequences of active TB. There is, however, a need to balance potential benefits against the risks and costs of screening. The $2013 \mathrm{WHO}$ recommendations on systematic screening for active TB state that indiscriminate mass screening should be avoided; while risk groups should be prioritised for screening based on careful assessment of the local TB epidemiology, the potential benefits and risks of harm of screening, and alternative interventions to improve early TB detection [3].

It is against this background that, on August 16-17, 2014, a group of Asian leaders from 14 countries, including national and international experts on diabetes and rheumatology, met in Macau, China to initiate discussion of the post-2015 WHO challenge of active case finding and prevention as a strategy for accelerating $\mathrm{TB}$ elimination in Asia. The objectives of the meeting were to discuss the strategy and evidence for active case finding and prevention among high-risk groups; review the current epidemiology and practices of Asian countries with respect to case finding; and to explore the most advanced approaches to investigate and treat contacts exposed to multidrug-resistant TB (MDR-TB), as well as screening and prevention for populations at risk in high-burden settings.

The meeting was sponsored and coordinated by Stop TB Partnership Japan and co-chaired by Toru Mori (Director Emeritus of Research Institute of Tuberculosis (RIT), Japan Anti-Tuberculosis Association (JATA)) and Erlina Burhan (Head of the Clinical Expert Team on MDR-TB, and Dept of Pulmonology and Respiratory Medicine, Faculty of Medicine, University of Indonesia).

The theme of the meeting was introduced by the keynote speaker, Nobu Nishikiori (Team leader, Stop TB and Leprosy Elimination, WHO Western Pacific Region (WPRO)), who provided an overview of the targets linked to the post-2015 strategy. This was followed by presentations from regional and international experts analysing the current TB control situation and assessing the feasibility of potential interventions in the context of scaling up active case finding and TB preventive treatment.

Paediatricians, rheumatologists and experts in diabetes all presented on the impact of TB in their field of expertise, clearly emphasising the importance of active case finding, better TB control and screening in select targeted groups. Although the heterogeneity of the region was widely recognised, an overarching consensus emerged on the need to identify and target high-risk populations within the context of country specific epidemiological and control settings. In particular, screening and active case finding interventions 


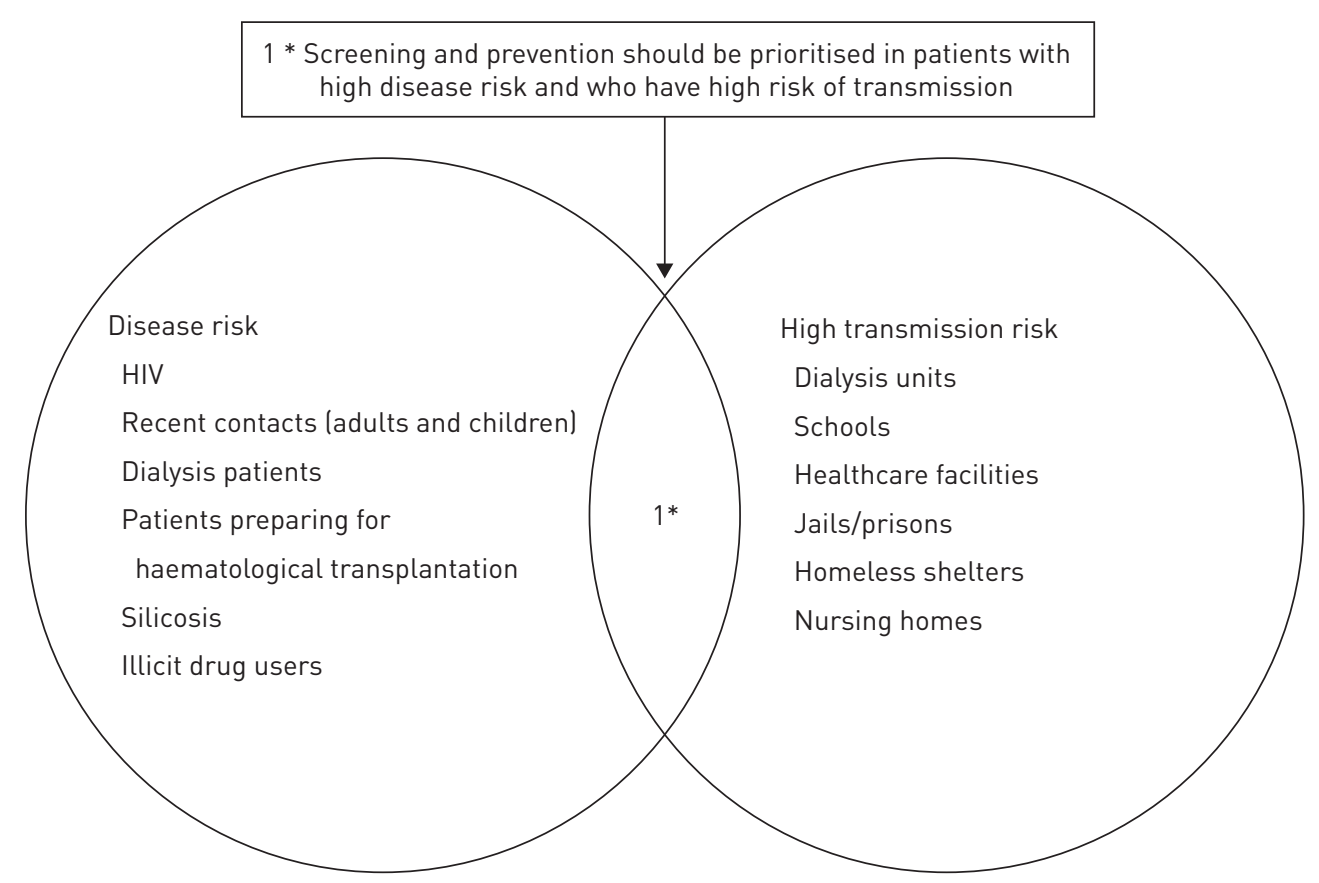

FIGURE 1 A hypothetical approach for prioritising selection of high-risk groups. Information from [3].

should become a matter of priority in those populations where the morbidity and mortality risk far exceeds that of the surrounding general populations [3]. There was unanimous agreement that without a scale up of these targeted active case finding interventions, it would be unlikely that any substantial progress towards TB elimination would be achieved in the decades to come. It became clear from several country and expert presentations that due consideration should now be given to expanding the role of preventive treatment as a programmatic intervention with harm-benefit and cost-effectiveness rationale taken into consideration in the different settings. Risk group prioritisation should take place on the basis of assessment of transmission and individual impact risk.

This meeting was convened in the spirit of advancing TB control in Asia and represents the first concerted regional response to the call for a change in pace made in the post-2015 strategy. Regional and country specific issues and approaches were discussed and debated in the spirit of reaching the highest ideals. Other regions have recognised the need to initiate dialogue in this direction, and have established forums and channels for intense discussion of the value of and need for solid TB elimination strategies [4-9].

Although an Asian regional consensus on the next steps requires further concerted action under national and regional leadership, the participants agreed their intention to advance acceleration towards elimination of TB in the following ways. 1) Better understanding, targeting and implementation of active case finding interventions with the aim of reaching out to the most affected in order to maximise the impact on the TB epidemic. 2) Considering and evaluating approaches for TB prevention as a strategy to eliminate TB in Asia and accelerate burden decline. 3) TB prevention needs to be tailored to the current epidemiological and TB control situation in countries while recognising the undeniable right of each individual to access a holistic spectrum of TB services, including TB prevention. 4) Recognising that concerted health infrastructure development, leadership, solidarity and action will be required to shape the future of TB control in Asia. was recognised http://ow.ly/LnWPW

Toru Mori ${ }^{1,2}$ and Erlina Burhan ${ }^{3}$ on behalf of participants in the third meeting of the Asian TB Experts Community ${ }^{4}$

${ }^{1}$ Stop TB Partnership Japan. ${ }^{2}$ Research Institute Tuberculosis (RIT) and Japan Anti-Tuberculosis Association (JATA), Tokyo, Japan. ${ }^{3}$ Dept of Pulmonology and Respiratory Medicine, Faculty of Medicine, University of Indonesia, Persahabatan Hospital Jakarta, Jakarta, Indonesia. ${ }^{4}$ A full list of participants in the third meeting of the Asian TB Experts Community and their affiliations can be found in the acknowledgements section.

Correspondence: Toru Mori, Research Institute of Tuberculosis, Japan Anti-Tuberculosis Association, 3-1-24, Matsuyama, Kiyose, Tokyo, 204-8533, Japan. E-mail: tmori-rit@jata.or.jp 
Support statement: Funding for the meeting was provided to STOP TB Japan through an unrestricted educational grant from QIAGEN. No promotional activity took place in the context of or associated with the meeting.

Conflict of interest: Disclosures can be found alongside the online version of this article at erj.ersjournals.com

Acknowledgements: The participants in the third meeting of the Asian TB Experts Community were as follows. Anis Karuniawati: Dept of Pulmonology and Respiratory Medicine, Persahabatan Hospital Jakarta, Faculty of Medicine, University of Indonesia, Jakarta, Indonesia; Arisu Kamada: Hokkaido Medical Center, Japan; Camilo Roa: Fellow Philippine College of Chest Physicians, Philippines; Chareon Chuchottaworn: Central Chest Institute of Health, Dept of Medical Services, Ministry of Public Health Thailand, Thailand; Darmawan Budi Setyanto: Division of Respirology, Pediatric Dept, RSCM hospital, Faculty of Medicine, University of Indonesia, Indonesia; Dyah Erti Mustikawati: National TB Program, Directorate DTDC, Ministry of Health Indonesia, Indonesia; Davide Manissero: Scientific and Medical Affairs, QIAGEN, UK; Erlina Burhan: Dept of Pulmonology and Respiratory Medicine, Persahabatan Hospital Jakarta, Faculty of Medicine, University of Indonesia, Jakarta, Indonesia; Fera Ibrahim: Dept of Pulmonology and Respiratory Medicine, Persahabatan Hospital Jakarta, Faculty of Medicine, University of Indonesia, Jakarta, Indonesia; Gan Suay Hong: Singapore TB Control Unit, Singapore; Gopalan Narendra: National Institute for Research in Tuberculosis, New Delhi, India; Guiyuan Jiang: Respiratory Dept, Xiamen Chang Gung Hospital, China; Hee Jin Kim: Korean Institute of Tuberculosis, Korea; Jianhua Zhu: Respiratory Dept, Nanfang Hospital, Nanfang Medical University, China; Jin Takasaki: National Center for Global Health and Medicine, Japan; Jing Wang: Laboratory dept, China-Japan Friendship Hospital, China; Joseph K.M. Kam: Centre for Health Protection, Dept of Health, Hong Kong; Kaewalee Soontornmon: Bureau of Tuberculosis, Dept of disease control, Ministry of Public Health Thailand, Thailand; Lei Gao: Institute of Pathogen Biology, Institute of Chinese academy of Medical Sciences and Peking Union Medical College, China; Lin Zhu: Tongji Medical College, Huazhong University of Science and Technology, China; Masae Loyce Kawamura: Scientific and Medical Affairs, QIAGEN, USA; Mengqiu Gao: TB Dept, Beijing Chest Hospital, Beijing, China; Nastiti Kaswandani: Division of Respirology, Pediatric Dept, RSCM hospital, Faculty of Medicine, University of Indonesia, Indonesia; Nobuyuki Nishikiori: Stop TB and Leprosy at WHO Manila, Philippines; Prakash Pispati: Rheumatology, Jaslok Hospital and Research Centre, and Saifee Hospital, Mumbai, India; Prijanti Soepandi: Dept of Pulmonology and Respiratory Medicine, Persahabatan Hospital Jakarta, Faculty of Medicine, University of Indonesia, Jakarta, Indonesia; Richard Brostrom: TB Control Program, Hawaii, USA; Ritesh Gupta: Fortis Centre of excellence for diabetes, metabolic disease and endocrinology, India; Satoshi Mitarai: Research Institute of Tuberculosis Japan, Japan; Seiya Kato: Research Institute of Tuberculosis Japan, Japan; Seung Chul Lee: Korea Centre of Disease Control, Korea; Shuihua Lu: TB Dept, Shanghai Public Clinical Health Center, China; Srikanth Tripathy: Jalma Agra, ICMR Institute, India; Toru Mori: Research Institute of Tuberculosis (RIT), Japan; Visalakshi Potharaju: Ampath Hyderabad, India; Wei Kwang Luk: Infection Control, Tseung Kwan Hospital, Taiwan; Wenjiang Ning: Laboratory Dept, Peking University Third Hospital, China; Zongde Zhang: Beijing Chest Hospital, China; Ying Meng: Respiratory Dept at Nanfang Hospital, China; Yunfeng Deng: Laboratory Dept, Shandong Chest Hospital, China; Zamzurina Abdul Bakar: Institute of Respiratory Medicine, Kuala Lumpur, Malaysia.

\section{References}

1 World Health Organization. Global strategy and targets for tuberculosis prevention, care and control after 2015. Geneva, World Health Organization, 2013. http://apps.who.int/gb/ebwha/pdf_files/EB134/B134_12-en.pdf?ua=1

2 World Health Organization. Guidelines on the management of latent tuberculosis infection. Geneva, World Health Organization, 2014.

3 World Health Organization. Systematic screening for active tuberculosis: principles and recommendations. Geneva, World Health Organization, 2013.

4 Migliori GB, Fleck F. Collaboration is key for new global tuberculosis strategy. Bull World Health Organ 2014; 92: 316-317.

5 Sotgiu G, Migliori GB. Is tuberculosis elimination a reality? Lancet Infect Dis 2014; 14: 364-365.

6 D’Ambrosio L, Dara M, Tadolini M, et al. Tuberculosis elimination: theory and practice in Europe. Eur Respir J 2014; 43: 1410-1420.

7 Diel R, Loddenkemper R, Sotgiu G, et al. Cost-effectiveness of treating latent tuberculous infection: a step towards elimination? Int J Tuberc Lung Dis 2013; 17: 1515.

8 Voniatis C, Migliori GB, Voniatis M, et al. Tuberculosis elimination: dream or reality? The case of Cyprus. Eur Respir J 2014; 44: 543-546.

9 Diel R, Loddenkemper R, Zellweger JP, et al. Old ideas to innovate tuberculosis control: preventive treatment to achieve elimination. Eur Respir J 2013; 42: 785-801. 